\title{
Das Selbstbelastungsverbot zugunsten juristischer Personen
}

\section{Tobias Weber *}

Während die strafrechtliche Verantwortlichkeit der juristischen Person im materiellen Recht bereits seit einiger Zeit etabliert ist, bleiben bezüglich der prozessualen Stellung von Unternehmen verschiedene Fragen offen. Der folgende Beitrag setzt sich mit der Frage auseinander, ob auch juristische Personen vor Selbstbelastungszwang zu schützen sind. In der Rechtsprechung wird der juristischen Person die Berufung auf den nemo-tenetur-Grundsatz zwar grundsätzlich gewährt, jedoch nicht ohne gleichzeitig den Anwendungsbereich einzuschränken. Der Autor hinterfragt diese Rechtsprechung und kommt zum Schluss, dass diese Gerichtspraxis als zu restriktiv zu beurteilen ist.

I. Ausgangslage

II. Der grundrechtliche Schutz vor Selbstbelastung .........................................44

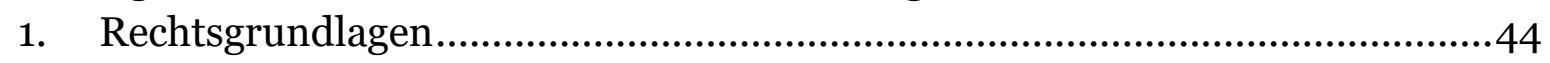

2. Anwendungsbereich .......................................................................44

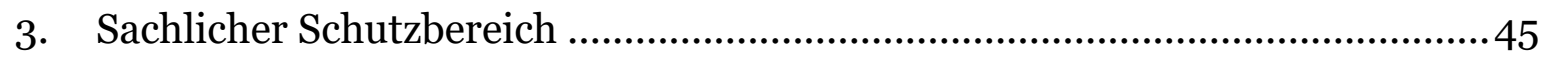

4. Bleibt Raum für Einschränkungen bei juristischen Personen? ......................4 47

III. Schutzzweck des Selbstbelastungsverbots ...................................................49

1. Zusammenhang zwischen Selbstbelastungsverbot und Unschuldsvermutung

2. Das Selbstbelastungsverbot als Ausfluss aus der Menschenwürde .................50

3. Das Selbstbelastungsverbot als Bestandteil eines fairen Verfahrens...............52

4. Die fehlende Menschenwürde als Einfallstor für Einschränkungen bei juristischen Personen? ................................................................... 55

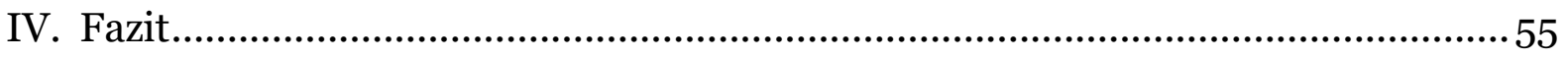

Zitiervorschlag: Tobias Weber, Das Selbstbelastungsverbot zugunsten juristischer Personen, in: sui-generis 2017, S. 42

URL: $\quad$ sui-generis.ch/32

DOI: $\quad$ https://doi.org/10.21257/sg.32

* Tobias Weber, MLaw, schloss sein Studium an der Universität Zürich im Herbst 2016 ab und absolviert derzeit ein Praktikum zur Vorbereitung auf die Anwaltsprüfung. Dieser Beitrag ist eine überarbeitete und gekürzte Fassung seiner Masterarbeit. 


\section{Ausgangslage}

1 Das Strafrecht war lange Zeit auf die individuelle Strafbarkeit natürlicher Personen ausgelegt. Heute ist allerdings bekannt, dass auch juristische Personen sowohl im Bereich des Kernstrafrechts als auch des Nebenstrafrechts Sanktionsrisiken ausgesetzt sind. ${ }^{1}$ Die Berufung auf etablierte strafprozessuale Mindestgarantien wird juristischen Personen zwar meist diskussionslos zuerkannt. ${ }^{2}$ Doch gerade beim Schutz vor Selbstbelastung scheint die Rechtsprechung nicht gewillt zu sein, die Mindestgarantie ohne Einschränkungen auf juristische Personen zu übertragen. 3 Dies, obwohl der Verfassungsrang bzw. die Grundrechtsqualität des Selbstbelastungsverbots heute in Lehre und Rechtsprechung fast einhellig anerkannt wird.4 Die von der Rechtspre-

1 Zu verweisen ist etwa auf Art. 102 StGB sowie die möglichen Sanktionen gegen juristische Personen im Nebenstrafrecht (z.B. Art. 7 VStrR, Art. 181 Abs. 1 DBG, Art. 57 Abs. 1 StHG oder Art. 49a KG).

2 Vgl. etwa die implizite Anerkennung der Unschuldsvermutung für juristische Personen in Urteil des Bundesgerichts 2C_290/2011 vom 12. September 2011, E. $5.1 \mathrm{ff}$.

3 BGE 142 IV 207 E. 8.3.3; BGE 140 II 384 E. 3.3.4 S. 393 .

4 BGE 130 I 126 E. 2.1 S. 128 m.w.H.; Urteil des EGMR 19187/91 vom 17. Dezember 1996 (Saunders gegen United Kingdom), Rn. 68; Auer Andreas/Malinverni Giorgio/Hottelier Michel, Droit constitutionnel suisse, Bd. II: Les droits fondamentaux, 3. Aufl., Bern 2013, Rz. 1351; Engler Marc, Die Vertretung des beschuldigten Unternehmens, Diss. Freiburg, Zürich 2008, S. 93; Flachsmann Stefan/Wehrenberg Stefan, Aussageverweigerungsrecht und Informationspflicht, SJZ 2001, S. 315; Ott Dominique, Der Grundsatz «nemo-tenetur se ipsum accusare: unter besonderer Berücksichtigung der strassenverkehrsrechtlichen Pflichten, Diss. Zürich, Zürich 2012, S. 175; Schlauri Regula, Das Verbot des Selbstbelastungszwangs im Strafverfahren., Diss. Zürich, Zürich 2003, S. 90 f.; a.M. Arzt Gunther, Schutz juristischer Personen gegen Selbstbelastung, JZ 2003, S. 456 ff.; Seiler Hansjörg, Das (Miss-) Verhältnis zwischen strafprozessualem Schweigerecht und verwaltungsrechtlicher Mitwirkungs- chung verordneten Einschränkungen des Selbstbelastungsverbots bei juristischen Personen lassen sich wie folgt zusammenfassen:

1. In der Rechtsprechung wird wiederholt ausgeführt, der sachliche Schutzbereich des Selbstbelastungsverbots sei bei juristischen Personen eng zu fassen. So würden insbesondere Unterlagen, die aufgrund von gesetzlichen Dokumentationspflichten erstellt wurden, nicht dem Selbstbelastungsverbot unterliegen. 5

2. Begründet wird diese restriktive Rechtsprechung damit, dass der nemo-tenetur-Grundsatz «bei natürlichen Personen (auch) einen Ausfluss aus der Menschenwürde» bilde und diese Komponente bei juristischen Personen fehle. 6 Gerade bei beschuldigten juristischen Personen sei deshalb «eine differenzierte $\mathrm{Ab}$ wägung vorzunehmen zwischen ihren grundrechtlich garantierten Verfahrensrechten und dem öffentlichen Interesse (sowie gegebenenfalls von geschädigten Personen) an einer effizienten Wahrheitserforschung.»7

2 Im vorliegenden Aufsatz soll die Gültigkeit dieser Einschränkungen überprüft werden. Dazu ist in einem ersten Teil der Gehalt des nemo-tenetur-Grundsatzes darzulegen. In einem zweiten Teil ist zu

und Auskunftspflicht, recht 2005, S. 20: «Die Auffassung, es gebe ein verfassungs- oder menschenrechtliches Schweigerecht, ist rational nicht begründbar und findet weder in der Verfassung noch in den Menschenrechtskonventionen eine Grundlage.»

5 BGE 142 IV 207 E. 8.3.3; BGE 140 II 384 E. 3.3.4 S. 393.

$6 \quad$ BGE 140 II 384 E. 3.3.4 S. 393.

7 BGE 142 IV 207 E. 8.4. 
prüfen, ob der Schutzzweck des Selbstbelastungsverbots Anpassungen bei juristischen Personen rechtfertigt.

\section{Der grundrechtliche Schutz vor Selbstbelastung}

\section{Rechtsgrundlagen}

3 Explizit verbürgt ist das Selbstbelastungsverbot lediglich in Art. 14 Abs. 3 lit. g UNO-Pakt II. Im Grundrechtskatalog der Bundesverfassung und der EMRK wird das Selbstbelastungsverbot dagegen nicht ausdrücklich erwähnt.

4 Nach aktueller Rechtsprechung ergibt sich das Selbstbelastungsverbot aus der Unschuldsvermutung (Art. 32 Abs. 1 BV) bzw. den Verteidigungsrechten der angeklagten Person (Art. 32 Abs. 2 BV), wobei offen bleiben kann, auf welcher der beiden Rechtsgrundlagen der nemo-teneturGrundsatz beruht. ${ }^{8}$ Das Bundesgericht verweist zudem regelmässig auf Art. 6 Ziff. 1 EMRK und die dazugehörige Praxis des EGMR. 9

Der EGMR leitet das Selbstbelastungsverbot in ständiger Rechtsprechung aus den Verfahrensgarantien gemäss Art. 6 Ziff. 1 EMRK ab, welche ein faires Verfahren gewährleisten sollen. ${ }^{10}$ Daneben verweist der Gerichtshof regelmässig auf den engen Zusammenhang zwischen dem Selbstbelastungsverbot und der in Art. 6 Ziff. 2 EMRK verankerten Unschuldsvermutung. ${ }^{11}$

8 BGE 131 I 272 E. 3.2.; BGE 130 I 126 E. 2.1 S. 129.

9 BGE 140 II 384 E. 3.3.2 S. 390; BGE 138 IV 47 E. 2.6.1 S. 51 .

10 Urteil des EGMR 19187/91 vom 17. Dezember 1996 (Saunders gegen United Kingdom), Rn. 68: «[...] the right to silence and the right not to incriminate oneself are generally recognized international standards which lie at the heart of the notion of a fair procedure under Article $6[\ldots]$ ».

11 Ebd.: «In this sense the [right not to incriminate

\section{Anwendungsbereich}

6 Die Anwendung der strafprozessualen Mindestgarantien gemäss EMRK und BV setzt eine strafrechtliche Anklage voraus, wobei der Begriff der strafrechtlichen Anklage vom EGMR autonom ausgelegt wird. ${ }^{12}$

7 Eine Anklage i.S.v. Art. 6 Ziff. 1 EMRK liegt nicht nur vor, wenn formell Anklage erhoben wurde, sondern auch wenn aufgrund der Umstände eine spätere Strafverfolgung als wahrscheinlich erscheint und der Betroffene deshalb als «substantially affected» zu betrachten ist. ${ }^{13}$ In einem reinen Verwaltungsverfahren fehlt in der Regel ein Bezug zu einer späteren Strafverfolgung. Der strafrechtliche Konnex wird aber dann hergestellt, wenn es wahrscheinlich erscheint, dass die in einem Verwaltungsverfahren erhobenen Beweismittel in einem nachfolgenden Sanktionsverfahren verwertet werden könnten. ${ }^{14}$

8 Eine Strafsache i.S.v. Art. 6 Ziff. 1 EMRK liegt vor, «wenn alternativ entweder das

oneself] is closely linked to the presumption of innocence.»

12 Urteil des EGMR 19187/91 vom 17. Dezember 1996, (Saunders gegen United Kingdom), Rn. 67. Auch das Bundesgericht orientiert sich bezüglich des Begriffs der strafrechtlichen Anklage an der Rechtsprechung des EGMR, vgl. BGE 135 I 313 E. 2.2.1.

13 Urteil des EGMR 34720/97 vom 21. Dezember 2000 (Heaney und McGuinness gegen Irland), Rn. 41 ff.; ähnlich BGE 106 Ia 7 E. 4 S. 9: «[Das Aussageverweigerungsrecht] gilt auch dann, wenn die Strafuntersuchung gegen den Betroffenen noch nicht formell eröffnet ist, aber unmittelbar bevorsteht.»; Ott (Fn. 4), S. 111.

14 Urteil des EGMR 31827/96 vom 3. Mai 2001, (J.B. gegen Schweiz), Rn. 66, wo es der Gerichtshof gar als ausreichend erachtete, dass der Beschwerdeführer nicht ausschliessen konnte, später wegen Steuerdelikten angeklagt zu werden; Benedick Gilles, Das Aussagedilemma in parallelen Verfahren, AJP 2011, S. 173; Ott (Fn. 4), S. 111 f. 
nationale Recht eine staatliche Massnahme dem Strafrecht zuordnet oder die Natur des Vergehens oder die Art und Schwere des Vergehens und/oder der Sanktionen für einen strafrechtlichen Charakter sprechen (sogenannte «Engel〉 -Kriterien).»15 In einem ersten Schritt ist folglich zu untersuchen, ob die in Frage stehende Norm nach Massgabe des innerstaatlichen Rechts dem Strafrecht zuzuordnen ist. Ist dies nicht der Fall, so ist nach der «wahren Natur», also dem Sinn und Zweck der entsprechenden Norm zu fragen. Richtet sich die Norm an jedermann und bezweckt sie vorwiegend Abschreckung und Vergeltung, so wird die Norm regelmässig als strafrechtlich qualifiziert. Verbleiben Unsicherheiten, so ist in einem letzten Schritt zu prüfen, ob die Schwere der drohenden Sanktion auf einen strafrechtlichen Charakter schliessen lässt. Ist eines der Kriterien erfüllt, so liegt eine Strafsache i.S.v. Art. 6 Ziff. 1 EMRK vor. ${ }^{16}$

9 Durch die weite Auslegung des Begriffs der Strafsache steht fest, dass die Verfahrensgarantien nach Art. 6 Ziff. 1 EMRK nicht nur bei kernstrafrechtlichen Verfahren zu gelten haben, sondern grundsätzlich auch in nebenstrafrechtlichen Verfahren zu beachten sind. In Bezug auf juristische Personen wurde dies bereits bei spielbankenrechtlichen sowie kartellrechtlichen Bussen nach Art. 51 SBG bzw. Art. 49a KG vom Bundesgericht bestätigt mit der Begründung, mit diesen Sanktionen werde nicht ein ausschliesslich prä-

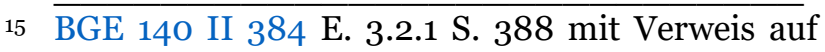
das Urteil des EGMR 5100/71 vom 8. Juni 1976 (Engel and others gegen The Netherlands).

16 Zum Ganzen Niggli Marcel Alexander/Maeder Stefan, Das Enforcementverfahren der Finanzmarktaufsicht (FINMA), Jusletter vom 7. März 2016, Rz. 6; Ott (Fn. 4), S. 108 ff. ventiver, sondern auch gleichzeitig ein pönaler bzw. repressiver Zweck verfolgt. Dieser komme darin zum Ausdruck, dass nicht bloss der erzielte Gewinn eingezogen, sondern zusätzlich auch ein Sanktionszuschlag verhängt werde. ${ }^{17}$ Die Anwendung der Verfahrensgarantien nach Art. 6 Ziff. 1 EMRK ist folglich immer dann zu prüfen, wenn die drohenden Massnahmen nicht als rein präventiv einzustufen sind bzw. nicht ausschliesslich auf die Wiederherstellung des ordnungsgemässen Zustandes oder den Entzug der durch die Pflichtverletzung erlangten Vorteile abzielen. ${ }^{18}$

\section{Sachlicher Schutzbereich}

10 Im Kern schützt der nemo-teneturGrundsatz den Beschuldigten vor Zwang zur Mitwirkung an seiner eigenen Überführung. ${ }^{19}$ Verboten sind also bestimmte Beweiserhebungsmethoden, die darauf abzielen, den Beschuldigten zur aktiven Mitwirkung an seiner eigenen Überführung $\mathrm{zu}$ zwingen. ${ }^{20} \mathrm{Im}$ Strafverfahren darf der Beschuldigte nicht zur Aussage gezwungen werden, was neben der An-

$1 7 \longdiv { \text { BGE } 1 4 0 \text { II } 3 8 4 \text { E. 3.2.2; vgl. BGE } 1 3 9 \text { I } 7 2 \text { E. 2.2 } }$ S. 79 .

18 Ausführlich dazu Häner Isabelle, Mindestgarantien für Strafverfahren und ihre Bedeutung für verwaltungsrechtliche Sanktionen, in: Häner Isabelle/Waldmann Bernhard (Hrsg.), Verwaltungsstrafrecht und sanktionierendes Verwaltungsrecht, Zürich 2010, S. 33 ff.

19 Urteil des Bundesgerichts 6B_503/2007 vom 21. Januar 2008, E. 3.3; Engler (Fn. 4), S. 92; Drope Katharina, Strafprozessuale Probleme bei der Einführung einer Verbandsstrafe, Diss. Hamburg, Berlin 2002, S. 150; Lutz Louise, Die Verteidigung und das Verbot, den Angeschuldigten zu seiner Selbstbelastung zu verpflichten, ZstrR 2012, 411; Ott (Fn. 4), S. 177 ff.

20 Wohlers Wolfgang, in: Donatsch Andreas/Hansjakob Thomas/Lieber Viktor (Hrsg.), Kommentar zur Schweizerischen Strafprozessordnung (StPO), 2. Aufl., Zürich 2014, Art. 3, Rz. 26; vgl. auch BGE 106 Ia 7 E. 4 S. 8. 
wendung körperlichen Zwangs auch das Androhen von Bussen oder anderen Sanktionen im Falle der Verweigerung verbietet. ${ }^{21}$ Der Beschuldigte muss seine Aussage verweigern können, ohne dass ihm daraus Nachteile erwachsen. ${ }^{22}$ Das Schweigen des Beschuldigten darf auch nicht als Indiz für dessen Schuld gewertet werden. ${ }^{23}$ Auf den Grund der Aussageverweigerung darf es nicht ankommen. ${ }^{24}$

11 Der Beschuldigte darf weiter nicht gezwungen werden, Beweisstücke, wie zum Beispiel Urkunden, herauszugeben (sog. Editionsverweigerungsrecht). 25 Aufgrund der Rechtsprechung des EGMR herrscht eine gewisse Unsicherheit, ob vorbestehende (‘pre-existing ) Beweismittel, wozu insbesondere bereits erstellte Dokumente zählen, auch vom Schutzbereich des nemo-tenetur-Grundsatzes erfasst werden. ${ }^{26}$ In Saunders $v$. UK hielt der

21 Wohlers Wolfgang, in: Donatsch/Hansjakob/ Lieber (Fn. 20), Art. 3, Rz. 28; vgl. auch Urteil des EGMR vom 34720/97 27. Dezember 2000, (Heaney and McGuiness gegen Irland), Rn. 51; Urteil des EGMR 18731/91 vom 8. Februar 1996, (John Murray gegen United Kingdom), Rn. 48 f.; Urteil des EGMR 19187/91 vom 17. Dezember 1996 (Saunders gegen United Kingdom), Rn. 69 f.

22 BGE 138 IV 47 E. 2.6.1 S. 51.

23 BGE 138 IV 47 E. 2.6.1 S. 51; BGE 131 IV 36 S. 41 E. 3.1 m.w.H.; gemäss EGMR darf hingegen das Schweigen des Beschuldigten unter gewissen Umständen zu dessen Nachteil ausgelegt werden, vgl. dazu Urteil des EGMR 18731/91 vom 8. Februar 1996 (John Murray gegen Vereinigtes Königreich), Rn. 47; kritisch dazu Graf Damian K., Schweigen ist Blei. Aussageverweigerung als Schuldindiz im Strafprozessrecht?, SJZ 2015, S. 193.

24 BGE 109 Ia 166 E. 2.b S. 168.

25 Urteil des EGMR 10828/84 vom 25. Februar 1993 (Funke gegen Frankreich), Rn. 44; Bertossa Carlo Antonio, Unternehmensstrafrecht - Strafprozess und Sanktionen, Diss. Basel, Bern 2003, S. 143; Engler (Fn. 4), S. 94; Meier Marcel, Strafrechtliche Unternehmenshaftung, Diss. Zürich, Zürich 2006, S. 148; Ott (Fn. 4), S. 188.

26 Ausführlich zur Kontroverse Ott (Fn. 4), S. 126 ff.
EGMR nämlich noch fest, dass die erzwungene Herausgabe von Beweismitteln, die unabhängig vom Willen des Beschuldigten existieren, nicht gegen das Selbstbelastungsverbot verstosse. ${ }^{27}$ Diese Rechtsprechung steht jedoch im Widerspruch zum früheren Entscheid Funke $v$. France $^{28}$ sowie den jüngeren Entscheiden J.B. v. Switzerland ${ }^{29}$ und Chambaz v. Switzerland $3^{\circ}$, wo der Gerichtshof in der erzwungenen Herausgabe von bereits bestehenden Dokumenten einen Verstoss gegen den nemo-tenetur-Grundsatz erblickte. ${ }^{31}$ In Übereinstimmung mit der jüngeren Rechtsprechung des EGMR nimmt die heute wohl herrschende Lehre an, dass auch die erzwungene Herausgabe von vorbestehenden Dokumenten gegen das Selbstbelastungsverbot verstösst. $3^{2}$

12 Die Verwertung von Beweismitteln, die ohne aktive Mitwirkung des Beschuldigten erhoben wurden, verletzt den nemo-

27 Urteil des EGMR 19187/91 vom 17. Dezember 1996 (Saunders gegen Vereinigtes Königreich), Rn. 74.

28 Urteil des EGMR 10828/84 vom 25. Februar 1993 (Funke gegen Frankreich).

29 Urteil des EGMR 31827/96 vom 3. Mai 2001, (J.B. gegen Schweiz).

3о Urteil des EGMR 11663/04 vom 5. April 2012, (Chambaz gegen Schweiz).

${ }^{31}$ Geth Christopher, Aufsichtsrechtliche Mitwirkungspflichten und nemo-tenetur, in: Emmenegger Susanne (Hrsg.), Banken zwischen Strafrecht und Aufsichtsrecht, Basel 2014, S. 155; Roth Simon, Das Verhältnis zwischen verwaltungsrechtlichen Mitwirkungspflichten und dem Grundsatz nemo-tenetur se ipsum accusare, ZStrR 2011, S. 314.

32 Geth, in: Emmengger (Fn. 31), S. 155; Kobel Pierre, Sanctions du droit des cartels et problèmes de droit administratif pénal, AJP 2004, S. 1156; Ott (Fn. 4), S. 129 f.; Roth (Fn. 31), S. 314 f.; Schlauri (Fn. 4), S. 171; vgl. auch Urteil (des Bundesverwaltungsgerichts) vom 24. Februar 2010, B-2050/ 2007 E. 5.7.4.1; a.M. Wohlers Wolfgang, in: Donatsch/Hansjakob/Lieber (Fn. 20), Art. 3, Rz. 29. 
tenetur-Grundsatz dagegen nicht.33 Den Beschuldigten trifft eine passive Duldungspflicht, d.h. er muss strafprozessuale Zwangsmassnahmen wie körperliche Durchsuchungen bzw. Untersuchungen, DNS-Analysen oder Beschlagnahmungen von Gegenständen und Vermögenswerten über sich ergehen lassen. 34 Eine unzulässige aktive Mitwirkungspflicht wird überall dort erblickt, wo die fraglichen Beweismittel durch eine «erzwungenen Willensbetätigung» des Beschuldigten erhoben wurden. 35

Die Unterscheidung zwischen aktiver Mitwirkung und passiver Duldung wird allerdings vermehrt in Frage gestellt. ${ }^{6}$ Dies insbesondere, weil der EGMR nicht konsequent zwischen aktiver Mitwirkung und passiver Duldung unterscheidet,37 sondern in Jalloh $v$. Germany eigene Prüfkriterien entwickelt hat.38 Ein Verstoss gegen das Selbstbelastungsverbot liegt gemäss der Rechtsprechung des EGMR nur vor, wenn die Mitwirkung durch ungerechtfertigten bzw. missbräuchlichen Zwang ( improper compulsion`) erreicht wurde. 39 Das Selbstbelastungsverbot gilt in diesem Sinne nicht

Benedick (Fn. 14), S. 172; Engler (Fn. 4), S. 94; vgl. auch Urteil des EGMR 10828/84 vom 25. Februar 1993 (Funke gegen Frankreich), Rn. 63 ff.

34 BGE 131 IV 36 E. 3.5.1; vgl. auch die Regelung in Art. 113 Abs. 1 Satz 2 StPO, dazu Engler Marc, in: Niggli Marcel Alexander/Heer Marianne/Wiprächtiger Hans (Hrsg.), Basler Kommentar Strafprozessordnung, 2. Aufl., Basel 2014, Art. 113, Rz. 8.

35 Lieber Viktor, in: Donatsch/Hansjakob/Lieber (Fn. 20), Art. 113, Rz. 44.

36 Ott (Fn. 4), S. 177 ff.; vgl. auch Lieber Viktor, in: Donatsch/Hansjakob/Lieber (Fn. 20), Art 113, Rz. 43a.

37 Wohlers Wolfgang, in: Donatsch/Hansjakob/Lieber (Fn. 19), Art. 3, Rz. 27.

38 Urteil des EGMR 54810/oo vom 11. Juli 2006 (Jalloh gegen Deutschland).

39 Urteil des EGMR 54810/oo vom 11. Juli 2006, (Jalloh gegen Deutschland), Rn. 100. absolut.40 Das Gericht berücksichtigt vielmehr «die Art und das Ausmass des ausgeübten Zwanges, das Bestehen von verfahrensrechtlichen Sicherungen und den Gebrauch, der von solcherart erlangten Beweisen gemacht wird.»41 Das Bundesgericht scheint sich in seiner jüngsten Rechtsprechung den vom EGMR aufgestellten Kriterien angleichen $\mathrm{zu}$ wollen. ${ }^{42}$ Besonders stark gewichtet das Bundesgericht dabei das erste Kriterium (Art und das Ausmass des angewandten Zwangs), während die weiteren Kriterien nur am Rande erwähnt werden.43 Dies hängt wohl auch damit zusammen, dass die Praxis des EGMR zu diesen Kriterien von Widersprüchen geprägt ist. 44

\section{Bleibt Raum für Einschränkungen bei juristischen Personen?}

14 In Bezug auf die eingangs erwähnten Bemühungen der Rechtsprechung, den Schutzbereich des Selbstbelastungsverbots bei juristischen Personen einzuengen muss bemerkt werden, dass zumindest bei gegen natürliche Personen gerichteten Sanktionsverfahren keine belastenden Dokumente unter Strafandro-

40 Ott (Fn. 4), S. 115 f. Nach Rechtsprechung des EGMR verstossen aktive Mitwirkungspflichten nicht per se gegen das Selbstbelastungsverbot. Siehe etwa Urteil des EGMR 15809/02 und 25624/O2 vom 11. Juli 2007, (O’Halleron and Francis gegen United Kingdom), wo kein Verstoss gegen das Selbstbelastungsverbot darin erblickt wurde, dass die Halter von geblitzten Fahrzeugen unter Strafandrohung verpflichtet wurden, Auskunft über die Lenker zu erteilen.

41 Ott (Fn. 4), S. 137; vgl. Urteil des EGMR 54810/oo vom 11. Juli 2006 (Jalloh gegen Deutschland), Rn. 101.

42 BGE 140 II 384 E. 3.3.3 S. 391.

43 BGE 140 II 384 E. 3.3.5 S. 393: «Entscheidend ist, dass keine «improper compulsion» ausgeübt wird, [...] wie dies der EGMR wiederholt formuliert hat.»

44 Ausführlich dazu Roth (Fn. 31), S. 315 ff. 
hung ediert werden dürfen.45 Da der EGMR in seinen Entscheiden zu Art. 6 Ziff. 1 EMRK, soweit ersichtlich, noch nie eine unterschiedliche Behandlung von natürlichen und juristischen Personen befürwortet hat, ist nicht davon auszugehen, dass der Gerichtshof zukünftig beim Schutzbereich des nemo-tenetur-Grundsatzes zwischen natürlichen und juristischen Personen differenzieren wird. Die Einschränkung, wonach gerade juristische Personen aufgrund von Dokumentationspflichten erstellte Unterlagen in einem Sanktionsverfahren herausverlangt werden können, scheint somit eine rein schweizerische Doktrin zu sein, die in der Rechtsprechung des EGMR jedenfalls bislang keine Stütze findet.

Zutreffend ist zudem auch die Feststellung, dass die Behörden, welche die Sanktionen gegen juristische Personen anordnen können, regelmässig nicht Adressaten der aufgrund von gesetzlichen Dokumentationspflichten erstellten Dokumente sind. ${ }^{46}$ Es ist zwar richtig, dass die Verwertung von unter Mitwirkungspflicht erhobenen Beweismitteln in einem reinen Verwaltungsverfahren unproblematisch ist. Sobald solche Beweismittel allerdings in einem Verfahren, das als strafrechtliche Anklage i.S.d. EGMR-Rechtsprechung zu qualifizieren

45 Urteil des EGMR 11663/04 vom 5. April 2012, (Chambaz gegen Schweiz), §§ 50 ff.

46 Tschudin Michael, Mitwirkungspflicht an der eigenen Sanktionierung, AJP 2016, S. 331, der zutreffend feststellt, dass im Kartellrecht keine Dokumentationspflichten existieren und folglich die Wettbewerbsbehörden auch nicht Adressaten der aufgrund von gesetzlichen Pflichten erstellten Unterlagen sind. Das gleiche gilt natürlich auch für ein von der Bundesanwaltschaft herausverlangtes Memorandum, das aufgrund von regulatorischen Pflichten zuhanden der FINMA erstellt wurde. Diese Tatsache wurde etwa in BGE 142 IV 207 nicht berücksichtigt. ist, verwertet werden sollen, muss der nemo-tenetur-Grundsatz greifen. $\mathrm{Zu}$ dieser Einsicht gelangte im Bereich des Steuerstrafrechts auch der Gesetzgeber. Als Reaktion auf Rügen des EGMR wurden dort die Art. 183 Abs. 1bis DBG sowie Art. 57a Abs. 2 StHG erlassen.47 Demnach dürfen im Steuerhinterziehungsverfahren, das strafrechtlicher Natur ist, Beweismittel, die in einem Nachsteuerverfahren unter Androhung einer Busse wegen Verletzung von Verfahrenspflichten beschafft wurden, nicht verwertet werden. Ob die Mitwirkung durch Strafandrohung unmittelbar erzwungen wird oder aufgrund von bestehenden Mitwirkungspflichten bloss mittelbar forciert wird, kann letztlich nicht massgebend sein. Andernfalls müsste juristischen Personen im Hinblick auf ein potentielles Sanktionsverfahren stets empfohlen werden, auch im Verwaltungsverfahren nie freiwillig zu kooperieren, sondern stets auf den Erlass einer strafbewehrten Verfügung zu beharren, mit der die Mitwirkung erzwungen wird.

16 Es sei zudem darauf hingewiesen, dass vorbestehende bzw. auf Grundlage von gesetzlichen Dokumentationspflichten erstellte Unterlagen in gegen juristische Personen geführten Sanktionsverfahren regelmässig die entscheidenden Beweismittel darstellen dürften. Schliesst man die Herausgabe solcher Dokumente vom sachlichen Schutzbereich des Selbstbelastungsverbots aus, so würde die Verfahrensstellung der juristischen Person erheblich geschwächt und das Selbstbelastungsverbot geradezu ausgehöhlt.

47 Vgl. Urteil des EGMR 11663/04 vom 5. April 2012 (Chambaz gegen Schweiz); Urteil des EGMR 31827/96 vom 3. Mai 2001 (J.B. gegen Schweiz). 
17 Es bleibt somit noch zu prüfen, ob die von der Rechtsprechung verordneten Einschränkungen des nemo-teneturGrundsatzes aufgrund der fehlenden Menschenwürde von juristischen Personen gerechtfertigt sind.

\section{Schutzzweck des Selbstbelastungs- verbots}

In Lehre und Rechtsprechung wurde über die Jahre eine kaum überblickbare Anzahl an Zweckbegründungen für den nemo-tenetur-Grundsatz herausgearbeitet. Es bietet sich deshalb an, die unterschiedlichen Begründungsansätze thematisch zu bündeln, wodurch sich im Wesentlichen drei unterschiedliche Argumentationslinien ergeben: die Herleitung des Selbstbelastungsverbots aus der Unschuldsvermutung, aus der Menschenwürde und aus dem fair-trialPrinzip.

\section{Zusammenhang zwischen Selbst- belastungsverbot und Unschulds- vermutung}

19 Ein Grossteil der Lehre sieht einen engen Zusammenhang zwischen der Unschuldsvermutung und dem Selbstbelastungsverbot. 48 Dieser wird etwa herge-

48 Auer/Malinverni/Hottelier (Fn. 4), Rz. 1351; Bertossa (Fn. 25), S. 143; Engler (Fn. 4), S. 93; Häner (Fn. 18), S. 23; Ott (Fn. 4), S. 175; Ruckstuhl Niklaus, Strafprozessuales Schweigerecht und verwaltungsrechtliche Mitwirkungsrespektive Auskunftspflicht - ein Gegensatz?, in: Schaffhauser René (Hrsg.), Jahrbuch zum Strassenverkehrsrecht 2006, St. Gallen 2006, S.225; Schlauri (Fn. 4), S. 98 ff.; Schneider Klaus, Unternehmensstrafbarkeit zwischen Obstruktion und Kooperation: strafrechtliche Begünstigung und Aussagedelikte im Rahmen der Strafbarkeit juristischer Personen nach Art. 102 StGB, Diss. Bern, Bern 2009, S. 77; Tophinke Esther, Das Grundrecht der Unschuldsvermutung, Diss. Bern, Bern 2000, S. 360 f.; Trechsel Stefan, Human stellt, indem argumentiert wird, das Selbstbelastungsverbot diene der Durchsetzung der Unschuldsvermutung. So wird geltend gemacht, der Stellenwert der Unschuldsvermutung in ihrer Ausprägung als Beweislastregel sänke, wenn der Beschuldigte zur Mitwirkung verpflichtet wäre.49 Die Unschuldsvermutung bliebe zwar formell bestehen, der Anklage würde es aber leichter fallen, die Schuld des Beschuldigten zu beweisen. ${ }^{50}$ Würde das Schweigen des Beschuldigten zu dessen Nachteil ausgelegt, so könnte allein durch das Beibringen von Indizien eine Erklärungspflicht des Beschuldigten entstehen, die sich zu seinen Lasten auswirkt, wenn er ihr nicht nachkommt. ${ }^{51}$ Umgekehrt wird auch angenommen, die Unschuldsvermutung diene der Durchsetzung des Selbstbelastungsverbots. Indem die Unschuldsvermutung den Beschuldigten von der Last befreie, seine Unschuld $\mathrm{zu}$ beweisen, diene sie als Grundlage für eine Verteidigung durch Schweigen.52 Weiter wird ausgeführt, dass die Unschuldsvermutung in ihrer Ausprägung als Beweiswürdigungsregel umgangen würde, wenn dem Beschuldigten für seine Mitwirkungsverweigerung eine Strafe drohen würde. Der Beschuldigte könnte so bestraft werden, obschon das Beweisergebnis in Bezug auf das ursprünglich verfolgte Delikt nicht für eine Verurteilung ausreichen würde und er gemäss dem Grundsatz in dubio pro reo freigesprochen werden müsste. 53

Rights in Criminal Proceedings, Oxford 2005, S. 348; a.M. Seiler (Fn. 4), S. 16.

49 Schlauri (Fn. 4), S. 99.

50 Schlauri (Fn. 4), S. 99; ähnlich Ruckstuhl (Fn. 48), S. 225.

51 Ruckstuhl (Fn. 48), S. 225.

52 Böse Martin, Die verfassungsrechtlichen Grundlagen des Satzes «Nemo-tenetur se ipsum accusare», GA 2002, S. $124 \mathrm{f}$.

53 Zum Ganzen Roth Simon, Zum Zweiten: Die Gel- 
20 Ein Zusammenhang zwischen der Unschuldsvermutung und dem Selbstbelastungsverbot ist durchaus erkennbar. Die Grundsätze können jedoch auch unabhängig voneinander bestehen bleiben. Ohne Geltung des nemo-teneturGrundsatzes könnte der Beschuldigte mittels Zwang zur Mitwirkung verpflichtet werden. Dies könnte einerseits durch direkten Zwang erfolgen, indem dem Beschuldigten im Falle einer Verletzung der Mitwirkungspflicht eine Strafe angedroht würde.54 Andererseits könnte die unberechtigte Verweigerung durch indirekten Zwang durchgesetzt werden, indem die Verweigerung der Mitwirkung bei der Beweiswürdigung negativ bewertet wird. 55 Die Unschuldsvermutung wäre sowohl bei direktem als auch indirektem Zwang zur Mitwirkung nicht unmittelbar beeinträchtigt. In beiden Fällen läge die Last für den Schuldbeweis immer noch beim Staat. Eine allfällige Bestrafung für die Mitwirkungsverweigerung bedeutet nicht, dass die Unschuldsvermutung im Verfahren, welches das ursprünglich angeklagte Delikt zum Gegenstand hatte, missachtet wurde. Denn in diesem Verfahren müsste bei mangelhafter Beweislage immer noch ein Freispruch gemäss dem Grundsatz in dubio pro reo erfolgen.

Wird der Beschuldigte zur Mitwirkung gezwungen, so verschiebt sich allerdings die Beweislast zum Nachteil des Beschuldigten. Kann die Mitwirkungsverweigerung negativ im Beweisergebnis berücksichtigt werden, gerät der Beschul-

tung von nemo-tenetur im Verwaltungsverfahren, Jusletter vom 24. November 2014, Rz. 46.

54 Wie etwa im Falle der unberechtigten Zeugnisverweigerung (Art. 176 StPO); Benedick (Fn. 14), S. 173.

55 Benedick (Fn. 14), S. 173; Seiler (Fn. 4), S. 10 mit Beispielen aus dem Verwaltungsrecht. digte unter Druck, seine Strategie des Schweigens aufzugeben und aktiv Beweise beizubringen, die seine Unschuld beweisen..$^{6}$ Im Falle der Durchsetzung mittels direkten Zwangs könnte der Staat zudem auf die Mitwirkung des Beschuldigten zählen. Verweigert der Beschuldigte seine Mitwirkung, obwohl ihm dafür eine Strafe droht, so könnte die verweigerte Mitwirkung zudem zu einem belastenden Indiz werden. Denn was könnte den Beschuldigten zur Verweigerung der Mitwirkung bewegen, obwohl ihm dafür eine Strafe droht? Ein rational handelnder Beschuldigter (bzw. ein anwaltlich gut beratener Klient) würde das kleinere Übel wählen. D.h. der Beschuldigte würde nur dann die Mitwirkung verweigern und die Strafe dafür in Kauf nehmen, wenn er dadurch verhindern könnte, eines gravierenderen Delikts überführt zu werden.

22 Der Schutzzweck des Selbstbelastungsverbots liegt folglich nicht allein in der Durchsetzung der Unschuldsvermutung. Die beiden Grundsätze ergänzen sich jedoch in einer Weise, dass der eine Grundsatz jeweils von der Existenz des Anderen abhängig ist. 57

\section{Das Selbstbelastungsverbot als Ausfluss aus der Menschenwürde}

23 In der Lehre werden regelmässig Begründungen des nemo-tenetur-Grundsatzes vorgebracht, die mit dem Schutz der Menschenwürde oder dem allgemeinen Persönlichkeitsrecht verbunden sind.

24 Mit Blick auf die historische Entwicklung wird etwa geltend gemacht, das Selbstbe-

56 Ruckstuhl (Fn. 48), S. 225.

57 Ebenso Böse (Fn. 52), S. 125; Ott (Fn. 4), S. 93; Schlauri (Fn. 4), S. 99. 
lastungsverbot schütze vor der Wiedereinführung der Folter und anderen Formen staatlicher Gewalt. $5^{8}$ Weiter wird argumentiert, der Beschuldigte stehe ohne Selbstbelastungsverbot vor einem unzumutbaren Dilemma: Er könne entweder sich selbst belasten oder müsse im Falle der Mitwirkungsverweigerung Zwangsmittel über sich ergehen lassen.59 Dadurch gerate der Beschuldigte in eine unzumutbare Entscheidungslage.60 Durch Zwang zur Selbstbelastung werde zudem die Willensfreiheit des Beschuldigten verletzt, was demütigend und entwürdigend sein könne. ${ }^{61}$ Ein Selbstbelastungszwang stehe zudem im Widerspruch zum natürlichen Selbsterhaltungstrieb. ${ }^{62}$ Der allgemeine Persönlichkeitsschutz verbiete es auch unabhängig einer allfälligen Verletzung der Menschenwürde, den Beschuldigten durch Redezwang einer unzumutbaren «Stresssituation» auszusetzen. ${ }^{63}$

Diese Ansätze vermögen nur sehr bedingt zu überzeugen. Es ist zunächst nicht davon auszugehen, dass sich der Staat zur Durchsetzung einer Pflicht zur Selbstbelastung grausamer Methoden bedienen würde. ${ }^{64}$ Das Verbot von Folter und unmenschlicher Behandlung ist ein eigenständiges Grundrecht, das unabhängig von einer allfälligen Pflicht zur

$58 \overline{\text { Lutz (Fn. 19), S. 415, wonach der nemo-tenetur- }}$ Grundsatz in erster Linie «die Abwehr finaler Zwangsausübung durch den Staat» bezwecke; Ott (Fn. 4), S. 74 m.w.H.

59 Ott (Fn. 4), S. 76; Schlauri (Fn. 4), S. 94; vgl. auch Trechsel Stefan, Bankgeheimnis - Steuerstrafverfahren - Menschenrechte. Nemo-tenetur bei Steuerhinterziehung, ZStrR 2005, S. 262 f.

60 Ruckstuhl (Fn. 48), S. 225; Schlauri (Fn. 4), S. 97 f.

61 Trechsel (Fn. 48), S. 348, der jedoch festhält, dass die Intensität der psychischen Belastung in der Regel nicht für eine Verletzung von Art. 3 EMRK ausreichen würde.

62 Ott (Fn. 4), S. 80 m.w.H.

63 Böse (Fn. 52), S. 104; Drope (Fn. 19), S. 199.

64 Ott (Fn. 4), S. 75.
Selbstbelastung zu beachten wäre. 65

26 Richtig ist dagegen, dass der Beschuldigte durch eine Pflicht zur Selbstbelastung in eine Zwangslage geraten würde. Auch seine Willensfreiheit würde in diesem Fall nicht respektiert. Ein seinem natürlichen Selbsterhaltungstrieb folgender Beschuldigter würde kaum aus freien Stücken an seiner eigenen Überführung mitwirken wollen. Dabei gilt es allerdings $\mathrm{zu}$ beachten, dass die passive Duldung von Zwangsmassnahmen auch nach geltendem Strafprozessrecht ganz selbstverständlich vom Beschuldigten verlangt wird, ohne dass der Willensfreiheit und dem natürlichen Selbsterhaltungstrieb des Beschuldigten Rechnung getragen wird. ${ }^{66}$ Inwiefern dies weniger unzumutbar ist als eine Verpflichtung zur aktiven Selbstbelastung, ist nicht klar ersichtlich. ${ }^{67}$ Es ist zum Beispiel nicht davon auszugehen, dass ein Beschuldigter aus freiem Willen seinen Mund zur Abgabe einer DNS-Probe öffnen würde, wenn er zu befürchten hätte, dadurch überführt zu werden. Auch der natürliche Selbsterhaltungstrieb würde die Abgabe einer potentiell belastenden DNS-Probe verbieten. Es kann also festgehalten werden, dass auch bei strafprozessualen Zwangsmassnahmen die Willensfreiheit und der natürliche Selbsterhaltungstrieb des Beschuldigten nicht respektiert wird. 68

Es stimmt zwar, dass der Beschuldigte das Ergebnis einer DNS-Probe nicht be-

65 Art. 10 Abs. 3 BV sowie Art. 3 EMRK.

66 Vgl. Art. 113 Abs. 1 Satz 2 StPO.

67 Kölbel Ralf, Selbstbelastungsfreiheiten. Der nemo-tenetur-Satz im materiellen Strafrecht, Habil. Jena, Berlin 2005, S. 285; Queck Nadine, Die Geltung des nemo-tenetur-Grundsatzes zugunsten von Unternehmen, Diss. Dresden, Berlin 2005, S. $151 \mathrm{ff}$.

68 Queck (Fn. 67), S. $151 \mathrm{ff}$. 
einflussen kann und somit nicht in eine «notstandsähnliche Entscheidungslage» gerät. ${ }^{69}$ Geht man aber davon aus, dass der nemo-tenetur-Grundsatz lediglich den Beschuldigten vor unzumutbaren Entscheidungslagen schützt, so müsste die Geltung des Grundsatzes je nach Schwere des Tatvorwurfs relativiert werden. Denn je schwerer der Tatvorwurf wiegt, desto stärker ausgeprägt wäre die psychische Beeinträchtigung durch Selbstbezichtigung.70 Die unzumutbare Entscheidungslage würde sich zudem vor allem dort akzentuieren, wo die verweigerte Mitwirkung mit direkten Sanktionen geahndet würde. Würde die verweigerte Mitwirkung bloss indirekt im Beweisergebnis berücksichtigt, wäre der innere Konflikt weniger stark ausgeprägt.

Zusammengefasst kann festgehalten werden, dass der Beschuldigte zwar durch einen Zwang zur Mitwirkung am eigenen Strafverfahren in eine Zwangslage geraten würde. Der Beschuldigte hat allerdings auch nach geltendem Prozessrecht Zwangsmassnahmen über sich ergehen zu lassen, ohne dass eine Verletzung der Menschenwürde oder ein Widerspruch zum natürlichen Selbsterhaltungstrieb geltend gemacht wird. Dass ein Verstoss gegen die Menschenwürde «im Spektrum aller strafprozessualer Inanspruchnahme» ausgerechnet beim Selbstbelastungszwang vorliegen soll, ist letztlich nur durch einen faktischen Konsens begründbar. ${ }^{71}$ Die Ratio des Selbstbelastungsverbots liegt folglich nicht im Schutz der Menschenwürde.

69 Ott (Fn. 4), S. 77 m.w.H.

70 Bosch Nikolaus, Aspekte des nemo-teneturPrinzips aus verfassungsrechtlicher und strafprozessualer Sicht, Diss. Augsburg, Berlin 1998, S. 33; Queck (Fn. 67), S. 149.

71 Kölbel (Fn. 67), S. 285.

\section{Das Selbstbelastungsverbot als Be- standteil eines fairen Verfahrens}

29 Unter dem Grundsatz des fairen Verfahrens werden in der Lehre verschiedene Aspekte eines rechtstaatlichen Verfahrens zusammengefasst.72 Der EGMR stützt sich bei der Auslegung des Grundsatzes auf die Präambel der EMRK, welche die Rechtsstaatlichkeit als übergeordnetes Ziel postuliert. Der nemotenetur-Grundsatz zählt gemäss der Rechtsprechung des EGMR zu den Kernelementen eines rechtstaatlichen und fairen Verfahrens nach Art. 6 Ziff. 1 EMRK.73 Auch das Bundesgericht nimmt bei der Begründung des Selbstbelastungsverbots Bezug auf den Grundsatz des fairen Verfahrens. 74

30 Unter dem Aspekt des fairen Verfahrens wird vertreten, das Selbstbelastungsverbot garantiere die «materielle Verteidigungsfreiheit» des Beschuldigten.75 Zwar bestehe ein öffentliches Interesse an der Wahrheitsfindung. ${ }^{76}$ Der Beschuldigte müsse aber frei sein, seine eigene Verteidigungsstrategie $\mathrm{zu}$ wählen und soll $\mathrm{zu}$ diesem Zweck mit angemessenen Mitwirkungsrechten ausgestattet werden.77 Müsste der Beschuldigte bereits von Beginn des Verfahrens bezüglich des Tatgeschehens aussagen, so wäre er im weiteren Verlauf des Verfahrens an seine Aussagen gebunden und könnte sich von seinen Äusserungen nicht mehr distanzieren, ohne an Glaubwürdigkeit zu verlieren. ${ }^{78}$

72 Vgl. Kölbel (Fn. 67), S. 299.

73 Exemplarisch: Urteil des EGMR 19187/91 vom 17. Dezember 1996 (Saunders gegen Vereinigtes Königreich), Rn. 68.

74 BGE 140 II 384 E. 3.3.5 S. 394; Urteil des Bundesgerichts 6B_439/2010 vom 29. Juni 2010, E. 5.2.

75 Böse (Fn. 52) S. 126 f.; Queck (Fn. 67), S. 183.

76 Queck (Fn. 67), S. 182.

77 Queck (Fn. 67), S. 183.

78 Queck (Fn. 67), S. 186. 
31 Das Selbstbelastungsverbot wird weiter aus dem Grundsatz der Waffengleichheit abgeleitet. Der Grundsatz der Waffengleichheit verlangt, dass der Beschuldigte im Strafverfahren dem Ankläger möglichst gleichgestellt ist. 79 Es soll also ein fairer Ausgleich zwischen der Anklagebehörde als Repräsentantin des Staates und dem Beschuldigten als Individuum hergestellt werden.80 Die machtvolle Stellung des Staates soll dadurch abgeschwächt werden, in dem der Beschuldigte mit Verteidigungsrechten ausgestattet wird. ${ }^{81} \mathrm{Zu}$ diesen Verteidigungsrechten gehöre u.a. das Selbstbelastungsverbot.82 Das Selbstbelastungsverbot verhindere eine "vorschnelle und einseitige Festlegung des Sachverhalts» durch die Untersuchungsbehörden. ${ }^{83}$ Das Selbstbelastungsverbot diene als Strukturprinzip, welches zusammen mit anderen, im Übergang vom Inquisitionsprozess zum reformierten Strafprozess entstandenen Verfahrensgrundsätzen, einen austarierten Strafprozess ermöglicht. Ein solcher Strafprozess diene aber nicht allein dem Ausgleich von Kollektiv- und Individualinteressen, sondern signalisiere der Öffentlichkeit, was der Beschuldigte dem Staat wert sei. ${ }^{84}$

79 Hauser Robert/Schweri Erhart/Hartmann Karl, Schweizerisches Strafprozessrecht, 6. Aufl., Winterthur/Horgen/Altdorf 2005, § 56, Rz. 17.

80 Flachsmann/Wehrenberg (Fn. 4), S. 315, die den Grundsatz des fairen Verfahrens in Art. 30 Abs. 1 BV und Art. 29 Abs. 1 BV verorten; vgl. auch Ott (Fn. 4), S. 94 m.w.H.

81 Flachsmann/Wehrenberg (Fn. 4), S. 315.

82 Flachsmann/Wehrenberg (Fn. 4), S. 315; aus dem Grundsatz der Waffengleichheit fliesst u.a. auch das rechtliche Gehör oder das Recht auf Verteidigung, vgl. dazu Hauser/Schweri/Hartmann (Fn. 79), § 56, Rz. 19.

83 Pieth Mark., Strafverfahren gegen Unternehmen, in: Arnold Jörg et al. (Hrsg.), Menschengerechtes Strafrecht: Festschrift für Albin Eser zum 70. Geburtstag, München 2005, S. 605.

84 Pieth (Fn. 83), S. 606 f.
32 Um zu verstehen, weshalb der nemotenetur-Grundsatz ein unverzichtbares Element eines fairen Verfahrens ist, lohnt sich ein Blick auf die Entwicklung des europäischen Strafverfahrens. Die Grundlagen des heute in Art. 6 Ziff. 1 EMRK verankerten fair-trial-Prinzips wurden im Übergang vom inquisitorischen Verfahren zum reformierten Strafprozess gelegt. ${ }^{85}$ Als Reaktion auf die Missbräuche im inquisitorischen Strafverfahren der frühen Neuzeit wurden Anklage und Richter personell getrennt und der Beschuldigte zusammen mit einem Rechtsbeistand unter dem Begriff der Verteidigung als Prozesspartei etabliert. ${ }^{86}$ Daraus resultierte die für den reformierten Strafprozess typische Dreiecksbeziehung zwischen Anklage und Verteidigung als Gegenspieler und dem Richter als unabhängige Entscheidungsinstanz. ${ }^{87}$ Die Verteidigung konnte nur als vollwertige Prozesspartei etabliert werden, indem man ihr gewisse Rechte zugestand. So war der Beschuldigte neu etwa berechtigt, am eigenen Strafverfahren $\mathrm{zu}$ partizipieren und es wurde ihm ein Verteidiger zur Seite gestellt. ${ }^{88}$ Die Stärkung der Verteidigung als Prozesspartei beschränkte sich jedoch vorerst auf die Hauptverhandlung, während der Beschuldigte im Vorverfahren nach wie

$85 \overline{\text { Summers Sarah J., Fair Trials. The European }}$ Criminal Procedural Tradition and The European Court of Human Rights, Diss. Zürich, Zürich 2006, S. 22 ff. In der Schweiz begann der Prozess um etwa 1830, vgl. dazu Hauser/Schweri/Hartmannn (Fn. 79), § 4, Rz. 12 ff.

86 Thommen Marc, Gerechtigkeit und Wahrheit im modernen Strafprozess, recht 2014, S. 266; zur Etablierung der Verteidigung als Prozesspartei Summers (Fn. 85), S. 75.

87 Summers (Fn. 85), S. 131, die feststellt, dass dieses System in den meisten europäischen Staaten gegen Ende des 19. Jh. etabliert war; Thommen (Fn. 86), S. 266.

88 Summers (Fn. 85), S. 67 ff., S. 84 ff. 
vor eine prekäre Stellung innehatte. ${ }^{89}$ Später wurde jedoch die Wichtigkeit des Vorverfahrens für den weiteren Ausgang des Prozesses erkannt und man gestand dem Beschuldigten im Sinne eines Ausgleichs das Recht zu, selbst über seine Mitwirkung entscheiden $\mathrm{zu}$ können.90 Von da aus entwickelte sich der nemotenetur-Grundsatz kontinuierlich weiter. 91

Der gedankliche Ursprung dieser Entwicklung lag nicht in der Erkenntnis, dass der Beschuldigte ein besonders schützenswertes Individuum darstellt und deshalb mit Verfahrensrechten ausgestattet werden muss. ${ }^{2}$ Man erkannte vielmehr, dass eine übermächtige Strafverfolgungsstelle zu Missbräuchen neigt und ihr deshalb ein Gegengewicht in Form der Verteidigung gegenübergestellt werden muss.93 Die neu gewonnenen Rechte des Beschuldigten waren folglich kein Selbstzweck, sondern dienten in erster Linie der Umsetzung des reformierten Strafprozesses, der einen Ausgleich zwischen Verteidigung und Anklage voraussetzte.94 Die Einsicht, dass ein Strafverfahren, indem der Beschuldigte mangels wirksamer Verteidigung von staatlicher

89

Eser Albin, Aussagefreiheit und Beistand des Verteidigers im Ermittlungsverfahren: Rechtsvergleichende Beobachtungen zur Rechtstellung des Beschuldigten, ZStW 1967, S. 568; Summers (Fn. 85), S. 93.

90 Summers (Fn. 85), S. 90, S. 163.

91 In der Schweiz etwa wurde im frühen 18. Jh. zunächst die Folter abgeschafft, später die Ungehorsams- und Lügenstrafen und erst im 20. Jh. die negativen Folgen der Aussageverweigerung. Ausführlich zur Entwicklung in der Schweiz Ott (Fn. 4), S. 43 ff.

92 Summers (Fn. 85), S. 75.

93 Ebd.

94 Summers (Fn. 85), S. 77: «[The participation of the accused] was seen to be a natural and essential consequence of the procedural reforms which dictated that there be a levelling of the roles of the defense and prosecution.»
Macht erdrückt wird, nicht legitim sein kann, war also schon früh ins Bewusstsein gelangt.

34 Diese Erkenntnis hat auch heute noch Geltung: Dem heutigen Strafprozessrecht liegt noch immer die Vorstellung des reformierten Strafprozesses zugrunde, der durch das Zusammenspiel von Verteidigung, Anklage und Richter funktioniert. 95 Es ist also auch heute noch ein ausbalanciertes Strafverfahrensrecht anzustreben, um den Beschuldigten vor einer übermächtigen Anklagebehörde zu schützen. Das Selbstbelastungsverbot ist freilich nur ein Teil dieses Ausgleichmechanismus und kann deshalb auch nicht isoliert betrachtet werden. Dem Ausgleich dienen auch die übrigen Rechte des Beschuldigten, insbesondere die Unschuldsvermutung, welche das Selbstbelastungsverbot quasi symbiotisch ergänzt. Dieser Ansatz verlangt auch nicht, dass die Anklagebehörde gänzlich entmachtet und jeder Eingriff in die Stellung des Beschuldigten als unzulässig erachtet wird. Denn dadurch würde wiederum die Balance zwischen Anklage und Verteidigung gestört. Den Strafverfolgungsbehörden steht eine ganze Reihe an Zwangsmassnahmen zur Verfügung, ohne die eine wirkungsvolle Strafverfolgung wohl illusorisch wäre. Diese hat der Beschuldigte aber nur zu erdulden, sofern die Voraussetzungen für die Ergreifung der konkreten Massnahme erfüllt sind. 96 Entscheidend für ein legitimes Verfahren

$95 \overline{\text { Dies gilt zumindest für das ordentliche Strafver- }}$ fahren. Etwas anders ist die Konstellation jedoch im heute verbreiteten Strafbefehlsverfahren (Art. 352 ff. StPO), wo man anstatt der klassischen Dreiecksbeziehung nur noch eine «Vertikale» bestehend aus Verteidigung und Staatsanwalt vorfindet. Dazu Thommen (Fn. 86), S. 273 ff.

96 Vgl. Art. 197 Abs. 1 StPO. 
ist also, dass der Beschuldigte durch wirksame Verteidigungsmöglichkeiten nicht einer übermächtigen Anklagebehörde ausgeliefert ist.

35 Der nemo-tenetur-Grundsatz kann somit in die verschiedenen vom Grundsatz des fairen Verfahrens erfassten Rechte des Beschuldigten eingereiht werden, welche in ihrer Gesamtheit einen legitimen Strafprozess bezwecken.

\section{Die fehlende Menschenwürde als Einfallstor für Einschränkungen bei juristischen Personen?}

36 Das Selbstbelastungsverbot bezweckt nicht wie von der Rechtsprechung angenommen den Schutz der Menschenwürde. Der Schutzzweck des nemo-teneturGrundsatzes kann vielmehr in der Wahrung eines fairen Verfahrens im weiteren Sinne verortet werden. Ein Strafverfahren ist dann als fair und legitim zu betrachten, wenn ein angemessener Ausgleich zwischen Anklage und Verteidigung besteht. Das Selbstbelastungsverbot ist Teil dieses Ausgleichs und schützt u.a. vor möglichen Übergriffen einer übermächtigen Untersuchungsbehörde. Der nemo-tenetur-Grundsatz steht in enger Verbindung mit den übrigen Verfahrensgarantien, wie etwa der Unschuldsvermutung. Das Selbstbelastungsverbot ist jedoch nicht bloss Ausfluss aus bestehenden Grundrechten, sondern ein eigenständiges Verfahrensgrundrecht, das zusammen mit weiteren Schutzbestimmungen zugunsten des Beschuldigten, ein faires und legitimes Strafverfahren garantieren soll.

37 Ein ausgeglichenes und waffengleiches Strafverfahren ist freilich ungeachtet der
Eigenschaften des Beschuldigten anzustreben. Das Selbstbelastungsverbot ist somit als verfassungsmässiges Recht vollumfänglich auf juristische Personen anwendbar. Nach dem Gesagten ist es auch nicht leicht einzusehen, warum gerade die Verfahrensrechte von juristischen Personen dem öffentlichen Interesse an einer effizienten Strafverfolgung untergeordnet werden sollen, zumal auch an einem legitimen Strafprozess ein öffentliches Interesse besteht. 97

\section{Fazit}

38 Der nemo-tenetur-Grundsatz ist immer dann zu beachten, wenn das Verfahren als strafrechtliche Anklage i.S. der EGMR-Rechtsprechung einzustufen ist. Dies kann sowohl bei kernstrafrechtlichen als auch bei neben- bzw. verwaltungsstrafrechtlichen Verfahren der Fall sein. In der Rechtsprechung des EGMR ist keine Lücke auffindbar, welche die vom Bundesgericht getroffenen Ausnahmen bei juristischen Personen betreffend vorbestehenden respektive aufgrund von gesetzlichen Dokumentationspflichten erstellten Unterlagen rechtfertigen würde.

39 Der Schutzzweck des Selbstbelastungsverbots kann in der Wahrung eines fairen Verfahrens im weiteren Sinne verortet werden kann. Der Grundsatz soll die staatliche Machtfülle im Strafverfahren eindämmen und einen fairen Ausgleich zwischen Anklage und Verteidigung gewährleisten. Da der nemo-tenetur-Grundsatz keine Verbindung zur Menschenwürde aufweist, gilt er auch zugunsten

$97 \overline{\text { Zum öffentlichen Interesse an einem legitimen }}$ Strafprozess vgl. Engler Marc, in: Niggli/Heer/ Wiprächtiger (Fn. 34), Art. 113, Rz. $3 a$. 
juristischer Personen vollumfänglich. Die juristische Person kann somit auch nicht aufgrund des hinter dem Grundsatz stehenden Schutzzweckgedankens gezwungen werden, inkriminierende Unterlagen herauszugeben. 